\title{
Constipation in chronic kidney disease: it is time to reconsider
}

\author{
Ryota Ikee ${ }^{1 *} \mathbb{D}$, Kazuhiro Yano ${ }^{1}$ and Tomomi Tsuru ${ }^{2}$
}

\begin{abstract}
Constipation is highly prevalent in patients with chronic kidney disease (CKD) and is primarily characterized by decreased intestinal motility. This chronic disorder affects the quality of life of patients. However, nephrologist and dialysis clinicians have long had a disproportionately limited understanding of constipation. Accumulating evidence has revealed a relationship between constipation and cardiovascular disease and CKD. The pathogenesis of constipation in CKD patients is multifactorial: decreased physical activity, comorbidities affecting bowel movement, such as diabetes mellitus, cerebrovascular disease, and hyperparathyroidism, a restricted dietary intake of plantbased fiber-rich foods, and multiple medications, including phosphate binders and potassium-binding resins, have all been implicated. CKD is associated with alterations in the composition and function of the gut microbiota, socalled gut dysbiosis. Recent studies showed that CKD-related gut dysbiosis decreased intestinal motility via intestinal inflammation or the increased generation of gut-derived uremic toxins, such as indoxyl sulfate and pcresyl sulfate. Furthermore, the gastrointestinal secretion of mucin was found to be decreased in CKD animal models, which may delay colonic transit by diminished lubrication in the alimentary tract. Thus, CKD-related gut dysbiosis may play a role in constipation, but limited information is currently available. Since constipation is often intractable, particularly in CKD patients, every available means needs to be employed in its treatment. The effects of probiotics, prebiotics, and synbiotics on the composition of the gut microbiota and gut-derived uremic toxins have been increasingly reported. However, their effects on stool consistency or frequency in CKD patients remain unclear. Some laxatives may be beneficial for improving not only bowel habits but also gut dysbiosis. Further studies are required to elucidate the CKD-specific pathogenesis of constipation and develop novel effective treatment options.
\end{abstract}

Keywords: Constipation, Chronic kidney disease, Dialysis, Uremic toxins, Intestinal motility, Gut microbiota, Gut dysbiosis, Chronic inflammation

\section{Background}

Constipation is a worldwide health issue and its median prevalence was reported to be $14 \%$ in adults overall and $33.5 \%$ in those aged over 60 years [1], with a higher susceptibility in females and individuals under a low socioeconomic status $[1,2]$. The chronic symptoms of constipation reduce the quality of life of patients as well as social activities $[1,3]$. Although substantial healthcare costs are consumed to treat this disorder, many patients are not satisfied with treatment because of the lack of efficacy [3]. Constipation often emerges as a secondary

\footnotetext{
*Correspondence: ryota.ikee@gmail.com

'Dialysis Vascular Access Center, Med.Co. LTA PS Clinic, 6-18 Tenyamachi,

Hakata-ku, Fukuoka, Fukuoka 812-0025, Japan

Full list of author information is available at the end of the article
}

manifestation of a number of diseases, including endocrine/metabolic diseases (diabetes mellitus [DM], hypothyroidism, hypercalcemia, and hyperparathyroidism), myopathic conditions (amyloidosis and scleroderma), neurological diseases (autonomic neuropathy, cerebrovascular disease, Parkinson's disease, and spinal cord injury), psychological conditions (depression and cognitive impairment), structural abnormalities (colonic and anal stricture, inflammatory bowel disease, and obstructive colonic mass), and chronic kidney disease (CKD) [1]. In a review by Murtagh et al., constipation was identified as the third most common symptom after fatigue and pruritus, with a prevalence of $57 \%$, among the various symptoms exhibited by patients with end-stage kidney disease (ESKD) [4]. Despite its high prevalence, nephrologists

(c) The Author(s). 2019 Open Access This article is distributed under the terms of the Creative Commons Attribution 4.0 International License (http://creativecommons.org/licenses/by/4.0/), which permits unrestricted use, distribution, and 
and dialysis clinicians have a limited understanding of constipation. In contrast to ischemic heart disease, stroke, and severe infection, constipation is not a life-threatening complication in CKD, except in rare cases of colonic bleeding due to stercoral ulcers [5]. However, several cohort studies have shown that constipation is associated with increased mortality in the general population [6], which may be explained by the relationship between constipation and colon cancer [7,8] and cardiovascular disease (CVD) [9-11]. Furthermore, a nationwide cohort study from the United States including 3 million veterans reported that constipation was associated with the higher incidence of CKD (hazard ratio, 1.13) and ESKD (hazard ratio, 1.09) [12]. More severe constipation was associated with the incrementally higher risk for each renal outcome. We have to change our prejudice that "constipation is not important in CKD." Although the pathogenesis of this complication is highly complex and multifactorial, accumulating evidence is prompting us to consider the relationship between constipation and CKD-related gut dysbiosis.

\section{Prevalence of constipation in CKD}

Chronic constipation is classified to three categories based on colonic transit and anorectal function [1]: normal transit constipation, slow transit constipation, and defecatory disorders. Normal transit constipation is the most common form encountered by clinicians [13]. Patients report symptoms that they consider to be consistent with constipation, such as hard stools or a perceived difficulty with evacuation. Most of these patients are treated empirically with dietary fiber or osmotic laxatives and responded well. Defecation disorders are a group of functional abnormalities of the pelvic floor or anorectum leading to the symptoms of constipation [13]. Slow transit constipation may be caused by dysfunctions in colonic smooth muscle or neural innervation, resulting in neural colonic motor abnormalities [14]. Wu et al. reported that the colonic transit time of patients treated with hemodialysis (HD) or peritoneal dialysis (PD) was significantly longer than that of age and sex-matched healthy subjects [15]. Delayed intestinal transit was also observed in CKD animal models [16-18]. Thus, constipation in CKD may primarily be classified as the slow transit type.

The reported prevalence of constipation widely ranges depending on the population investigated and how constipation is defined. If constipation is defined by the subjects themselves, its prevalence is higher, whereas it is more likely to be low based on objective definitions [2]. The definition of constipation differs between healthcare professionals and patients; the former generally define constipation based on stool frequency, whereas the latter define it as straining, hard stools, difficulty in passing stools, discomfort with defecation, or the feeling of incomplete evacuation [19, 20]. The Rome diagnostic criteria, which are expert consensus criteria for diagnosing functional gastrointestinal disorders, are a standardized, widely used method. The newest Rome IV criteria, released in May 2016, define functional constipation as shown in Table 1 [2]. According to these criteria, the diagnosis of constipation needs to be made after the exclusion of irritable bowel syndrome with predominant constipation (IBS-C). The basis of functional constipation is considered to involve delayed colonic transit and a failure to relax the pelvic floor muscles during defecation, whereas the pathophysiology of IBS-C involves a disorder in the brain-gut interaction with the predominant symptom of abdominal pain [21]. The differentiation of these disorders depends on the prominence of abdominal pain in IBS-C, which may be challenging in clinical practice. Actually, previous studies showed not only a diagnostic overlap [21-23] but also the migration of patients between these diagnoses over time [21]. Therefore, some authorities posed a question regarding the feasibility of the Rome criteria for the diagnosis of constipation [1, 24]. The Bristol Stool Form Scale (BSFS) [25], shown in Table 2, is another method

Table 1 Rome IV diagnostic criteria for functional constipation and irritable bowel syndrome with constipation

\footnotetext{
Diagnostic criteria for functional constipation $^{a}$

1. Must include 2 or more of the following

a. Straining during more than $25 \%$ of defecations

b. Lumpy or hard stools (the Bristol Stool Form Scale $\left.{ }^{c} 1-2\right)$ more

than $25 \%$ of defecations

c. Sensation of incomplete evacuation more than $25 \%$ of defecations

d. Sensations of anorectal obstruction/blockage more than 25\% of defecations

e. Manual maneuvers to facilitate more than $25 \%$ of defecations (e.g., digital evacuation, support of the pelvic floor)

f. Fewer than 3 spontaneous bowel movements per week

2. Loose stools are rarely present without the use of laxatives

3. Insufficient criteria for irritable bowel syndrome

Diagnostic criteria for irritable bowel syndrome with predominant constipation

1. Recurrent abdominal pain, on average, at least 1 day per week in

the last 3 months, associated with 2 or more of the following criteria:

a. Related to defecation

b. Associated with a change in frequency of stool

c. Associated with a change in form (appearance) of stool

2. More than $25 \%$ of bowel movement with Bristol stool form type

or 2 , and less than $25 \%$ of bowel movement with Bristol stool form type 6 or 7

${ }^{a}$ Criteria fulfilled for the last 3 months with symptom onset at least 6 months prior to diagnosis

${ }^{\mathrm{b}}$ For research studies, patients meeting criteria for opioid-induced constipation should not be given a diagnosis of functional constipation because it is difficult to distinguish between opioid side effects and other causes of constipation. However, clinicians recognize that these 2 conditions might overlap

'The Bristol Stool Form Scale is shown in Table 2

${ }^{d}$ Irritable bowel syndrome subtype can only be confidently established when the patient is evaluated off medication used to treat bowel habit abnormalities
} 
Table 2 Bristol Stool Form Scale

\begin{tabular}{ll}
\hline Type & Stool form \\
\hline 1 & Separate hard lumps, like nuts (hard to pass) \\
2 & Sausage-shaped but lumpy \\
3 & Like a sausage but with cracks on the surface \\
4 & Like a sausage or snake, smooth and soft \\
5 & Soft blobs with clear-cut edges \\
6 & Fluffy pieces with ragged edges, a mushy stool \\
7 & Watery, no solid pieces, entirely liquid \\
\hline
\end{tabular}

that is often used to estimate constipation. The BSFS reflects the colonic transit time: stool forms 1 and 2 are associated with slower transit, and stool forms 6 and 7 with more rapid transit [26]. The functions of the colon are to absorb water and electrolytes and transport waste to the rectum. Under a physiological state, the colon receives approximately $1.5 \mathrm{~L}$ of liquid effluent daily from the small intestine, with $200-400 \mathrm{~mL}$ being excreted in stools [13]. The removal of water from fecal slurry is time-dependent and actively regulated. Sodium is reabsorbed from luminal contents through several active transport channels, with water following passively in response to osmotic gradients. On the other hand, colonic secretion is mediated through chloride channels, which are generally quiescent, leading to a net absorption of water and electrolytes [13]. Thus, stools that remain in the colon longer become drier and harder. However, laxatives are regularly administered to a substantial proportion of CKD patients $[15,27,28]$ and strongly affect stool frequency and consistency. The cessation of laxatives in order to diagnose constipation is troublesome in clinical practice.

Tables 3 and 4 show the prevalence of constipation reported in nondialysis-CKD patients [29, 30] and dialysis patients [15, 27-29, 31-36], respectively. Cano et al. reported a higher prevalence of constipation in dialysis patients in a comparison with age and sex-matched community subjects $(31.1 \%$ vs. $10.1 \%, P<0.001)$ [32]. In dialysis patients, the definition/criteria of constipation are varied and its prevalence widely ranged between 14.2 and $71.7 \%$. The Rome criteria and BSFS have only been employed in a few studies. Regarding nondialysis-CKD patients, limited data are available on the prevalence of constipation [29, 30]. Further studies are required to clarify whether constipation increasingly occurs in proportion with the progression of CKD. The incidence of constipation after the induction of dialysis therapy is another important issue. Previous studies consistently reported that the prevalence of constipation was lower in PD patients than in HD patients [15, 29, 32, $34,35]$, even with matching for age, sex, and dialysis duration [35]. A dialysis modality-based lifestyle, higher dietary fiber intake, and smaller amounts of potassium-binding resins and phosphate binders may explain this lower prevalence of constipation in PD patients. In PD, however, clinicians should pay attention to constipation because of its association with peritonitis [37].

\section{Potential factors inducing constipation in CKD}

A vast number of factors are vital for normal intestinal motility, such as the nervous system (central, enteric, and autonomic), immune system, endocrine system, bile acid metabolism, mucus secretion, gut microbiota, and products of intestinal fermentation [14]. Disturbances in any of these systems may contribute to the symptoms of constipation. In the setting of CKD, the pathogenesis of constipation is more complex and multifactorial. Discussions on the potential pathogenic link between CKD-related gut dysbiosis and constipation/intestinal dysmotility are of importance.

\section{Gut dysbiosis}

Gut dysbiosis, which refers to alterations in the composition and function of the gut microbiota, has been attracting increasing attention due to its association with various diseases, including obesity, atherosclerosis, DM, cancer, and CKD [38]. Dietary components that escape digestion in the small intestine are fermented through metabolism by the gut microbiota. There are two main pathways of fermentation: saccharolytic and proteolytic fermentations. Saccharolytic fermentation is more favorable because of the beneficial metabolites produced, such as short-chain fatty acids (SCFA, acetates, propionates, and butyrates). In proteolytic fermentation, ammonia, amines, and a number of uremic toxin precursors (thiols, phenols, and indoles) are generated. The relationship between CKD and gut

Table 3 Prevalence of constipation in patients with nondialysis-CKD

\begin{tabular}{|c|c|c|c|c|}
\hline Authors (Year), country & No. of subjects & Age (year) & Renal function & Definition of constipation (Prevalence [\%]) \\
\hline Yasuda et al. (2002), Japan [29] & 105 & $59.1 \pm 11.9$ & Unknown & $\begin{array}{l}\text { Meeting } 1 \text { and 2, or } 3 \text { (59.9) } \\
\text { 1. Stool consistency: moderate or severe } \\
\text { 2. Straining: always or almost always } \\
\text { 3. Bowel movements }<3 \text { times/week }\end{array}$ \\
\hline Ramos et al. (2019), Brazil [30] & 43 & $59.0 \pm 13.5$ & eGFR $21.3 \pm 7.9 \mathrm{~mL} / \mathrm{min} / 1.73 \mathrm{~m}^{2}$ & $\begin{array}{l}\text { Rome III criteria (34.9) } \\
\text { BSFS type } 1 \text { and } 2 \text { (32.6) }\end{array}$ \\
\hline
\end{tabular}


Table 4 Prevalence of constipation in dialysis patients

\begin{tabular}{|c|c|c|c|c|}
\hline Authors (Year), country & No. of subjects & Age (year) & Dialysis duration & Definition of constipation (Prevalence [\%]) \\
\hline Hammer et al. (1998), Austria [31] & HD, 105 & Unknown & Unknown & $\begin{array}{l}\text { Straining, hard stool, or bowel } \\
\text { movements }<3 \text { times/week (40.0) }\end{array}$ \\
\hline Yasuda et al. (2002), Japan [29] & $H D, 268 ; P D, 204$ & $\begin{array}{l}H D, 56.4 \pm 11.7 \\
P D, 50.0 \pm 13.7\end{array}$ & Unknown & $\begin{array}{l}\text { Meeting } 1 \text { and 2, or } 3 \text { (HD, 63.1; PD, 28.9) } \\
\text { 1. Stool consistency: moderate or severe } \\
\text { 2. Straining: always or almost always } \\
\text { 3. Bowel movements < } 3 \text { times/week }\end{array}$ \\
\hline Wu et al. (2004), Taiwan [15] & $H D, 56 ; P D, 63$ & $\begin{array}{l}H D, 53.1 \pm 10.6 \\
P D, 50.3 \pm 11.0\end{array}$ & Unknown & Laxative use (HD, 35.7; PD, 15.9) \\
\hline Cano et al. (2007), United Kingdom [32] & $H D, 100 ; P D, 48$ & Unknown & Unknown & $\begin{array}{l}\text { Meeting at least } 2 \text { of the following symptoms } \\
\text { (HD, 33.0; PD 27.1) } \\
\text { 1. Bowel movements < } 3 \text { times/week } \\
\text { 2. Hard or lumpy stool } \\
\text { 3. Straining } \\
\text { 4. Feeling of incomplete emptying } \\
\text { 5. Sensation that the stool cannot be passed } \\
\text { 6. Need of manual maneuvers }\end{array}$ \\
\hline Bossola et al (2011), Italy [33] & $H D, 110$ & $64.6 \pm 14.8$ & $6.5 \pm 6.3$ years & Self-defined (27.3) \\
\hline Hatakeyama et al (2013), Japan [27] & HD, 112 & $60.2 \pm 10.8$ & $11.6 \pm 6.5$ years & Laxative use (27.7) \\
\hline Zhang et al. (2013), China [34] & $H D, 478 ; P D, 127$ & $\begin{array}{l}H D, 53.0 \pm 14.2 \\
P D, 45.2 \pm 13.1\end{array}$ & $\begin{array}{l}\text { HD, } 53.4 \pm 14.9 \text { months } \\
\text { PD, } 49.6 \pm 10.4 \text { months }\end{array}$ & Rome III criteria (HD, 71.7; PD, 14.2) \\
\hline Dong et al. (2014), China [35] & $\mathrm{HD}, 182 ; \mathrm{PD}, 112$ & $\begin{array}{l}H D, 58.7 \pm 14.4 \\
P D, 59.7 \pm 14.2\end{array}$ & $\begin{array}{l}\text { HD, } 55.5 \pm 38.5 \text { months } \\
\mathrm{PD}, 48.9 \pm 31.0 \text { months }\end{array}$ & $\begin{array}{l}\text { Gastrointestinal Symptom Rating Scale } \\
(H D, 36.3 ; P D, 17.9)\end{array}$ \\
\hline Ramos et al. (2015), Brazil [36] & $H D, 290$ & Unknown & Unknown & Rome III criteria (32.8) \\
\hline Ikee et al. (2016), Japan [28] & $H D, 136$ & $67 \pm 12$ & $103 \pm 103$ months & Laxative use (66.2) \\
\hline
\end{tabular}

$H D$ hemodialysis, $P D$ peritoneal dialysis

dysbiosis is widely recognized and involved in the concept of the gut-kidney axis. In this setting, the contraction of bacterial families possessing SCFA-forming enzymes and the expansion of families possessing urease, uricase, and indole- and p-cresol-forming enzymes occurs, and microbial metabolism consequently shifts to a predominantly proteolytic fermentation pattern due to uremiarelated biochemical changes in the gut environment [39]. Gut-derived uremic toxins, such as indoxyl sulfate (IS), p-cresyl sulfate (PCS), and trimethylamine- $\mathrm{N}$-oxide (TMAO), accumulate in the bodies of CKD patients via their increased generation as well as decreased renal excretion. These toxins induce oxidative stress and proinflammatory responses $[39,40]$. On the other hand, since butyrates promote the expression of tight junction proteins in the gut epithelium [41], the contraction of SFCAproducing bacteria impairs the integrity of the gut epithelium and results in the translocation of lipopolysaccharides in the circulation. Thus, gut dysbiosis is one of the main causes of chronic inflammation in CKD, which leads to the progression of CVD $[40,42,43]$ and CKD itself [40, 42] and strongly influences patient morbidity and mortality [44]. It is notable that gut dysbiosis was observed even in patients with mild CKD with estimated glomerular filtration rate of $76 \pm 15 \mathrm{~mL} / \mathrm{min} / 1.73 \mathrm{~m}^{2}$ [45]. In kidney transplantation, the gut microbiota may be associated with graft survival and its composition was altered by transplantation [46].
A recent experimental study by Hoibian et al. suggested a mechanism that links CKD-related gut dysbiosis to constipation [16]. In this study, adenine-induced CKD mice showed a 1.8-fold longer gastrointestinal transit time than the control mice. Resected colons from control mice were then incubated with the plasma of healthy subjects or HD patients. Colons incubated with uremic plasma exhibited a blunted level of contraction from those incubated with healthy plasma. Colons were subsequently incubated with uremic toxins at the concentration encountered in ESKD patients. The incubation with IS or PCS decreased the maximal force of colonic contractions. These findings demonstrated that some uremic toxins impair intestinal motility. Ramos et al. supported this hypothesis by a clinical study including 43 nondiabetic, nondialysis-CKD patients [30]. In this study, constipation defined by BSFS type 1-2 stools was associated with an increased level of serum PCS independently of renal function and dietary fiber intake. Decreased intestinal motility may impact uremic toxin generation by increasing the availability of amino acids to be fermented in the colon [30]. Accumulated uremic toxins, in turn, affect intestinal motility, thereby forming a vicious cycle.

Another pathogenic factor that potentially links CKDrelated gut dysbiosis to constipation may be intestinal inflammation. Yu et al. showed that intestinal motility in CKD rats underwent 5/6 nephrectomy was significantly 
decreased compared with that in the control rats [17]. The expression levels of interleukin-6, tumor necrosis factor- $\alpha$, and inducible nitric oxide synthesis were increased in the gut of uremic rats. Using the same animal models, Nishiyama et al. showed that uremia induced gut dysbiosis, intestinal dysmotility, a decreased amount of feces, as well as intestinal inflammation [18]. Of note, antibiotic therapy improved intestinal dysmotility, fecal amounts, and the expression of cytokines. In addition, they reported that in an ex vivo procedure similar to Hoibian's study, an incubation with spermine had a negative impact on intestinal motility, whereas that with IS did not [18]. This discordance may be attributable to the difference in IS concentration and in the incubation time.

Attempts to identify the pathogenic factors linking CKD-related gut dysbiosis to constipation have only just begun. Limited information is currently available on this issue. However, these attempts deserve more attention because they may contribute to the development of novel treatment options for constipation in CKD and additionally lead to the suppression of CKD and CVD.

\section{Lifestyle}

Population-based surveys have reported a relationship between decreased physical activity and constipation [1]. Physical activity is lower in dialysis patients than in healthy subjects [47, 48], and markedly lower in older patients [47]. Lower activity was observed even during interdialytic periods [48]. Moreover, Yasuda et al. reported that $78.5 \%$ of HD patients suppressed the urge to defecate during HD sessions [29]. Furthermore, many HD patients use laxatives every other day, not every day, to avoid defecation during HD sessions. These findings may partly explain the high prevalence of constipation in HD patients.

\section{Comorbidities}

Various comorbidities that induce secondary constipation, such as DM, autonomic neuropathy, and cerebrovascular disease, are common in CKD patients. Although DM is recognized to cause constipation [1], a substantial body of evidence negates this association $[49,50]$. Sommers et al. recently reported that the prevalence of constipation defined by BSFS type 1-2 stools was similar between diabetic and nondiabetic subjects in a nationally representative sample of adults in the USA [50]. Of note, impaired kidney function was more frequent in diabetic subjects with constipatoin than in those without constipation. On the other hand, Yamada et al. showed that peripheral neuropathy of the lower extremities correlated with the symptoms of constipation, irrespective of the presence of diabetic nephropathy or retinopathy [51]. In our previous study including $136 \mathrm{HD}$ patients, DM was independently associated with constipation defined by laxative use [28].
Although primary hyperparathyroidism causes constipation [52], to the best of our knowledge, the influence of secondary hyperparathyroidism on constipation has not yet been investigated.

\section{Medications}

A large number of medications, such as serotonin (5hydroxytriptamine [5-HT]) receptor blockers, opioids, anticholinergic agents, anticonvulsants, antihypertensive agents, antidepressants, and chemotherapy agents, may cause constipation [1]. Regarding drug-induced constipation, difficulties are associated with identifying the causal drug in CKD patients who receive many types of medications. Although reducing drugs potentially inducing constipation is essential in the management of constipation, it may be difficult in some patients with multiple severe complications. However, potassiumbinding resins and phosphate binders may more frequently induce constipation than other drugs. A previous study reported that the relative risk of constipation was 1.32 in HD patients taking potassium-binding resins relative to those not taking these agents [29]. Among phosphate binders, sevelamer appears to more frequently induce constipation [53]. Sevelamer is known to improve lipid profiles via the adsorption of bile acids [54, 55]. Since bile acids increase propagated colonic contractions and the secretion of water and electrolytes in the colon [56], sevelamer-induced constipation may be partly attributed to bile acid adsorption by the drug itself. Ironcontaining phosphate binders, such as sucroferric oxyhydroxide and ferric citrate, may induce diarrhea instead of constipation [57, 58]. It is important to note that iron supplementation may have a favorable or unfavorable influence on the gut microbiota [59-61].

\section{Dietary restrictions}

The intake of fiber-rich vegetables and fruits is restricted in order to inhibit hyperkalemia in patients with advanced $C K D$, particularly in $\mathrm{HD}$ patients. The mean value of dietary fiber intake previously reported in $\mathrm{HD}$ patients ranged between 5.9 and 16.6 g/day [29, 62-65]. In the general population, some epidemiological studies supported a relationship between low fiber intake and constipation [1]. Soluble fiber may accelerate intestinal transit via hydrophilic properties and the osmotic effects of fermentation by-products. Insoluble fiber accelerates transit by increasing the stool biomass, leading to the direct stimulation of secretion and motility [2]. However, the effects of dietary fiber therapy on constipation have been shown to depend on the category of constipation. According to a clinical study by Voderholzer et al., 85\% of patients with normal transit constipation showed the attenuation of symptoms following a dietary fiber treatment, whereas $63 \%$ of those with slow transit 
constipation did not respond to this therapy [66]. Considering these findings, the relationship between a reduced fiber intake and constipation may not be so sufficient in CKD patients. On the other hand, fiber intake is associated with reduced cardiovascular and cancer mortality [67]. Moreover, the benefits of a plant-based diet for CKD patients have been increasingly reported [68]. Further studies are required to more clearly demonstrate the benefits of dietary fiber as a main source of prebiotics.

Decreased fluid intake has been implicated as a cause of constipation in dialysis patients. However, we previously reported no significant differences in interdialytic weight gain between patients with and without constipation [28]. Wu et al. showed that interdialytic weight gain correlated with a longer colonic transit time [15]. It may be conceived that greater fluid intake may soften stools or enhance lubrication in the alimentary tract, but mucin is far more effective for lubrication. Decreased mucin secretion and the lower viscosity of gastric secretions have been reported in non-CKD patients with constipation, which may delay colon transit due to diminished lubrication [69]. It is important to note that the decreased secretion of mucin has also been observed in CKD rats, and this was increased by the administration of butyrates [70].

\section{Oxidative stress}

Oxidative stress has been suggested to play a role in colonic motor dysfunction in DM [71, 72]. DM represents a state of oxidative stress as a result of the hyperglycemiainduced generation of reactive oxygen species. Using specimens obtained by intestinal biopsy and colectomy from diabetic subjects, Chandrasekharan et al. demonstrated the loss of enteric neurons induced by increased oxidative stress [71]. As described above, gut-derived uremic toxins have the potential to activate oxidative stress [39, 40], which may lead to decreased intestinal motility.

\section{Hyperhomocysteinemia}

Homocysteine is an intermediate in methionine metabolism, and its plasma level increases in ESKD patients. Hyperhomocysteinemia has been suggested to contribute to the increased risk of CVD in this population [73]. In an experimental study by Givvimani et al., cystathionine $\beta$-synthase heterozygous knockout mice, well-known models of hyperhomocysteinemia, showed decreased intestinal motility [74]. Consistent with this finding, we reported that the increased plasma level of total homocysteine in HD patients was independently associated with constipation after adjustments for age, sex, and DM [28].

\section{Possible interventions for constipation in CKD}

Constipation in CKD is challenging to treat successfully due to its multifactorial nature. Therefore, every possible approach to ameliorate constipation needs to be considered. The expected goals of the following interventions may include: regular bowel movement, improvement of stool continence and incomplete evacuation, amelioration of gut microbiota composition, and decreased synthesis of harmful uremic toxins.

\section{Probiotics, prebiotics, and synbiotics}

Since gut dysbiosis is suggested to be associated with constipation, agents favorably affecting the growth or activity of beneficial microbiota may be a promising option for the treatment of constipation. Probiotics are live microorganisms that confer health benefits on the host. Prebiotics are indigestible food ingredients that include dietary fiber, oligosaccharides, polysaccharides, and resistant starches. Synbiotics refer to a combination of probiotics and prebiotics. A meta-analysis by Dimidi et al. revealed that probiotics reduced the whole-gut transit time in non-CKD patients by 12 hours and increased stool frequency by 1.3 times/week [75]. Similarly, prebiotics and synbiotics improved stool frequency and consistency [76]. On the other hand, limited information is currently available on their effects on bowel habits in CKD patients. Nakabayashi et al. reported that a synbiotic treatment for 2 weeks significantly increased stool quantities in HD patients [77]. Furthermore, hard stools appeared to soften. In nondialysis-CKD patients, fiber supplementation significantly increased stool frequency from $1.4 \pm 0.2$ to $1.9 \pm 0.3$ times/day [78]. In both studies, bowel habits were estimated as a secondary outcome. Further studies are required to examine the effects of these agents on stool frequency, stool consistency, and intestinal motility in CKD patients.

The effects of these agents on the composition of the gut microbiota and gut-derived uremic toxins have been increasingly reported. Based on a meta-analysis by McFarlane et al. [79], Mafra et al. did not recommend probiotics as a sole intervention, but suggested an increased intake of foods with a high content of prebiotics in CKD patients [38]. Further studies are required to establish a more effective, sophisticated prescription of these agents.

\section{Laxatives}

Osmotic laxatives are widely used in CKD, but their effect may be insufficient as a sole therapy in a substantial proportion of patients, particularly in ESKD patients. In these patients, Mg-containing laxatives may induce hazardous hypermagnesemia [80, 81]. Bulk-forming laxatives and polyethylene glycol have to be taken with a relatively large amount of water. Therefore, they are 
mainly treated with stimulant laxatives. This class of laxative stimulates peristalsis by directly irritating the smooth muscle of the colon and also increases fluid secretion. Progressive increases in the doses of these agents may be attributable to hypo-functioning in the bowel process, the loss of intrinsic innervation action, and laxative tolerance effects [82]. On the other hand, controversy exists regarding the tolerance effects and structural or functional alterations of the colon induced by stimulant laxatives $[83,84]$. It currently remains unclear whether the long-term use of stimulant laxatives is safe and reasonable. The American Gastroenterological Association mentioned that stimulant laxatives may be used as "rescue" agents in cases resistant to osmotic laxatives [1].

As a novel class of laxatives, intestinal secretagogues, such as lubiprostone, linaclotide, elobixibat, and tenapanor, recently exhibit efficacy against chronic constipation $[85,86]$. Lubiprostone is a chloride channel activator that increases water secretion into the intestinal lumen and enhances colonic transit. In HD patients, lubiprostone was reported to increase stool frequency significantly from $1.8 \pm 1.3$ to $4.5 \pm 1.5$ times/week [87]. It is notable that this agent increased gastric and intestinal mucin secretion $[88,89]$, potentially leading to lubrication of the alimentary tract $[69,89]$. In an experimental study using adenine-induced CKD mice, lubiprostone improved the composition of the gut microbiota and decreased plasma IS levels [90]. Additionally, lubiprostone exerted favorable effects on the gut epithelial barrier [91, 92]. Thus, lubiprostone may ameliorate not only constipation but also gut dysbiosis and epithelial barrier disruption in CKD patients. Elobixibat is an ileal bile acid transporter inhibitor that blocks bile acid absorption in the ileum and increases the delivery of bile acids into the colon. As described above, bile acids cause fluid secretion and enhance colonic motor activity [56]. Gen et al. reported a decrease in serum phosphate after lubiprostone therapy [87]. Accelerated intestinal transit may reduce phosphate absorption in the small intestine. Tenapanor, an inhibitor of sodium/hydrogen exchanger 3 , reduces the absorption of sodium and phosphate in the intestine. This agent may be preferred due to its phosphate-lowering effects.

Mineral oil acts as a lubricant and stool-softening laxative. Ramos et al. examined the effects of olive oil and flaxseed oil in HD patients with constipation and reported increased stool frequency and better consistency [36]. The effects and tolerability of oil administration warrant further study.

\section{Serotonin type 4 receptor agonists}

Serotonin type $4\left(5-\mathrm{HT}_{4}\right)$ receptor agonists stimulate peristalsis and accelerate gastrointestinal transit. Shin et al. reviewed 13 randomized controlled trials on highly selective $5-\mathrm{HT}_{4}$ receptor agonists in adults with chronic constipation and concluded that these drugs improved stool frequency and other symptoms of constipation, generally with minor neurological/psychiatric adverse events [93]. However, the efficacy of these drugs has not yet been reported in CKD/ESKD patients. Since CKDrelated constipation is often refractory, $5-\mathrm{HT}_{4}$ receptor agonists may be used not as a sole therapy but as an additive therapy to other drugs.

\section{Lifestyle-based therapy}

In a recent meta-analysis, Gao et al. suggested that exercise, such as walking, may be a feasible and effective strategy to ameliorate constipation-related symptoms and quality of life in the non-CKD population [94]. To the best of our knowledge, it currently remains unknown whether exercise interventions have a similar effect in CKD/ESKD patients. However, exercise therapy was recently suggested to improve physical function $[95,96]$ and other symptoms, including restless legs syndrome [97], in dialysis patients. Even if the effects of exercise on constipation are not sufficient, habitual exercise is associated with other beneficial effects.

Modulation of the defecation posture may be another option for constipation. During defecation, the squatting position facilitates rectal emptying by anorectal angle straightening, resulting in higher rectal pressure and lower anal pressure with relaxation of the levator ani [98]. Sikirov reported that the squatting position led to quicker evacuation and a more complete sense of bowel emptying than the sitting position [99]. However, most populations in developed countries have become accustomed to sitting on toilet seat. This custom may induce suboptimal bowel habits, particularly in older individuals with reduced straining and pelvic floor weakness. Modi et al. reported that the use of a footstool (defecation posture modification device) provided similar benefits to squatting [98].

These options may be adjunctive approaches at best for CKD patients with constipation, but may be performed safely and inexpensively.

\section{Conclusions}

Constipation is often intractable because its pathogenesis is multifactorial, particularly in CKD patients. Accumulating evidence has underlined the potential involvement of CKD-related gut dysbiosis in this complication. Pharmacological, dietary, and lifestyle-based approaches are applicable for its treatment, but their effects have not been fully investigated to date. In addition, appropriate management of constipation may be different according to CKD stage or modality of renal replacement therapy. As described above, CKD-related gut dysbiosis, uremic toxin accumulation, and intestinal dysmotility 
worsen in a vicious cycle manner. Treatment of gut dysbiosis in early stages of CKD may be desirable to inhibit their intractable aggravation. Further studies are required on this issue.

Elucidation of the relationship between CKD-related gut dysbiosis and constipation may contribute to the development of novel treatment options for constipation, which may also exert favorable effects on CKD and CVD.

\section{Abbreviations}

BSFS: Bristol Stool Form Scale; CKD: Chronic kidney disease;

CVD: Cardiovascular disease; DM: Diabetes mellitus; ESKD: End-stage kidney disease; HD: Hemodialysis; IBS-C: Irritable bowel syndrome with predominant constipation; IS: Indoxyl sulfate; PCS: p-Cresyl sulfate; PD: Peritoneal dialysis; SCFA: Short-chain fatty acid; TMAO: Trimethylamine-N-oxide; 5-HT: 5Hydroxytryptamine

\section{Acknowledgements}

Not applicable.

\section{Authors' contributions}

$\mathrm{Rl}, \mathrm{KY}$, and $\mathrm{TT}$ contributed to the research idea and study design. RI wrote the whole manuscript, and $\mathrm{KY}$ and $T \mathrm{~T}$ read and advised about the content of the manuscript of the final version. All authors read and approved the final manuscript.

\section{Funding}

Not applicable.

\section{Availability of data and materials \\ Not applicable.}

Ethics approval and consent to participate

Not applicable.

\section{Consent for publication}

Not applicable.

\section{Competing interests}

The authors declare that they have no competing interests.

\section{Author details}

'Dialysis Vascular Access Center, Med.Co. LTA PS Clinic, 6-18 Tenyamachi, Hakata-ku, Fukuoka, Fukuoka 812-0025, Japan. ${ }^{2}$ Department of Rheumatology, Med.Co. LTA PS Clinic, 6-18 Tenyamachi, Hakata-ku, Fukuoka, Fukuoka 812-0025, Japan.

Received: 23 July 2019 Accepted: 20 November 2019

Published online: 12 December 2019

\section{References}

1. Bharucha AE, Pemberton JH, Locke GR III. American Gastroenterological Association technical review on constipation. Gastroenterology. 2013;144: 218-38.

2. Lacy BE, Mearin F, Chang L, Chey WD, Lembo AJ, Simren M, et al. Bowel disorders. Gastroenterology. 2016;150:1393-407.

3. Johanson JF, Kralstein J. Chronic constipation: a survey of the patient perspective. Aliment Pharmacol Ther. 2007;25:599-608.

4. Murtagh $\mathrm{FE}$, Addington-Hall J, Higginson IJ. The prevalence of symptoms in end-stage renal disease: a systematic review. Adv Chronic Kidney Dis. 2007; 14:82-99.

5. Saeed F, Kalra A, Kousar N, Pace LA, Holley JL. Stercoral ulcer as a cause of lower gastrointestinal (LGI) bleeding in chronic hemodialysis patients. Clin Nephrol. 2012;77:75-8.

6. Chang JY, Locke GR, McNally MA, Halder SL, Schleck CD, Zinsmeister AR, et al. Impact of functional gastrointestinal disorders on survival in the community. Am J Gastroenterol. 2010;105:822-32.
7. Watanabe T, Nakaya N, Kurashima K, Kuriyama S, Tsubono Y, Tsuji I. Constipation, laxative use and risk of colorectal cancer: the Miyagi cohort study. Eur J Cancer. 2004;40:2109-15.

8. Guerin A, Mody R, Fok B, Lasch KL, Zhou Z, Wu EQ, et al. Risk of developing colorectal cancer and benign colorectal neoplasm in patients with chronic constipation. Aliment Pharmacol Ther. 2014;40:83-92.

9. Salmoirago-Blotcher E, Crawford S, Jackson E, Ockene J, Ockene I. Constipation and risk of cardiovascular disease among postmenopausal women. Am J Med. 2011;124:714-23.

10. Honkura K, Tomata Y, Sugiyama K, Kaiho Y, Watanabe T, Zhang S, et al. Defecation frequency and cardiovascular disease mortality in Japan: the Ohsaki cohort study. Atherosclerosis. 2016;246:251-6.

11. Kubota Y, Iso H, Tamakoshi A. Bowel movement frequency, laxative use, and mortality from coronary heart disease and stroke among Japanese men and women: the Japan collaborative cohort (JACC) study. J Epidemiol. 2016;26: 242-8.

12. Sumida K, Molnar MZ, Potukuchi PK, Thomas F, Lu JL, Matsushita K, et al. Constipation and incident CKD. J Am Soc Nephrol. 2017;28:1248-58.

13. Andrews CN, Storr M. The pathophysiology of chronic constipation. Can J Gastroenterol. 2011;25(Suppl B):16B-21B.

14. Dimidi E, Christodoulides S, Scott SM, Whelan K. Mechanisms of action of probiotics and the gastrointestinal microbiota on gut motility and constipation. Adv Nutr. 2017:8:484-94

15. Wu MJ, Chang CS, Cheng CH, Chen CH, Lee WC, Hsu YH, et al. Colonic transit time in long-term dialysis patients. Am J Kidney Dis. 2004;44:322-7.

16. Hoibian E, Florens N, Koppe L, Vidal H, Soulage CO. Distal colon motor dysfunction in mice with chronic kidney disease: putative role of uremic toxins. Toxins (Basel). 2018;10:204.

17. Yu C, Tan S, Wang Z, Deng B, Li J, Wang Q, et al. Chronic kidney disease elicits an intestinal inflammation resulting in intestinal dysmotility associated with the activation of inducible nitric oxide synthesis in rat. Digestion. 2018; 97:205-11.

18. Nishiyama K, Aono K, Fujimoto Y, Kuwamura M, Okada T, Tokumoto H, et al. Chronic kidney disease after 5/6 nephrectomy disturbs the intestinal microbiota and alters intestinal motility. J Cell Physiol. 2019;234:6667-78.

19. Connell AM, Hilton C, Irvine G, Lennard-Jones JE, Misiewicz JJ. Variation of bowel habit in two population samples. BMJ. 1965;2:1095-9.

20. Herz MJ, Kahan E, Zalevski S, Aframian R, Kuznitz D, Reichman S. Constipation: a different entity for patients and doctors. Fam Pract. 1996;13: 156-9.

21. Wong RK, Palsson OS, Turner MJ, Levy RL, Feld AD, von Korff M, et al. Inability of the Rome III criteria to distinguish functional constipation from constipation-subtype irritable bowel syndrome. Am J Gastroenterol. 2010; 105:2228-34.

22. Zhao YF, Ma XQ, Wang R, Yan XY, Li ZS, Zou DW, et al. Epidemiology of functional constipation and comparison with constipation-predominant irritable bowel syndrome: the systematic investigation of gastrointestinal diseases in China (SILC). Aliment Pharmacol Ther. 2011;34:1020-9.

23. Shekhar C, Monaghan PJ, Morris J, Issa B, Whorwell PJ, Keevil B, et al. Rome III functional constipation and irritable bowel syndrome with constipation are similar disorders within a spectrum of sensitization, regulated by serotonin. Gastroenterology. 2013;145:749-57.

24. Quigley EM. Editorial: differentiating chronic idiopathic constipation from constipation-predominant irritable bowel syndrome-possible and important? Aliment Pharmacol Ther. 2015:41:1299.

25. O'Donnell LJ, Virjee J, Heaton KW. Detection of pseudodiarrhoea by simple clinical assessment of intestinal transit rate. BMJ. 1990;300:439-40.

26. Lewis SJ, Heaton KW. Stool form scale as a useful guide to intestinal transit time. Scand J Gastroenterol. 1997;32:920-4.

27. Hatakeyama S, Murasawa H, Narita T, Oikawa M, Fujita N, Iwamura $H$, et al. Switching hemodialysis patients from sevelamer hydrochloride to bixalomer: a single-center, non-randomized analysis of efficacy and effects on gastrointestinal symptoms and metabolic acidosis. BMC Nephrol. 2013; $14: 222$.

28. Ikee R, Toyoyama T, Endo T, Tsunoda M, Hashimoto N. Clinical factors associated with constipation in hemodialysis patients. Int Urol Nephrol. 2016:48:1741-2.

29. Yasuda G, Shibata K, Takizawa T, Ikeda Y, Tokita Y, Umemura S, et al. Prevalence of constipation in continuous ambulatory peritoneal dialysis patients and comparison with hemodialysis patients. Am J Kidney Dis. 2002; 39:1292-9. 
30. Ramos Cl, Armani RG, Canziani ME, Ribeiro Dolenga CJ, Nakao LS, Campbell KL, et al. Bowel habits and the association with uremic toxins in non-dialysisdependent chronic kidney disease patients. J Ren Nutr. 2019 (in press)

31. Hammer J, Oesterreicher C, Hammer K, Koch U, Traindl O, Kovarik J. Chronic gastrointestinal symptoms in hemodialysis patients. Wien Klin Wochenschr. 1998;110:287-91.

32. Cano AE, Neil AK, Kang JY, Barnabas A, Eastwood JB, Nelson SR, et al. Gastrointestinal symptoms in patients with end-stage renal disease undergoing treatment by hemodialysis or peritoneal dialysis. Am J Gastroenterol. 2007;102:1990-7.

33. Bossola M, Luciani G, Rosa F, Tazza L. Appetite and gastrointestinal symptoms in chronic hemodialysis patients. J Ren Nutr. 2011;21:448-54.

34. Zhang J, Huang C, Li Y, Chen J, Shen F, Yao Q, et al. Health-related quality of life in dialysis patients with constipation: a cross-sectional study. Patient Prefer Adherence. 2013;7:589-94.

35. Dong R, Guo ZY, Ding JR, Zhou YY, Wu H. Gastrointestinal symptoms: a comparison between patients undergoing peritoneal dialysis and hemodialysis. World J Gastroenterol. 2014;20:11370-5.

36. Ramos Cl, Andrade de Lima AF, Grilli DG, Cuppari L. The short-term effects of olive oil and flaxseed oil for the treatment of constipation in hemodialysis patients. J Ren Nutr. 2015;25:50-6.

37. Su CY, Pei J, Lu XH, Tang W, Wang T. Gastrointestinal symptoms predict peritonitis rates in CAPD patients. Clin Nephrol. 2012;77:267-74.

38. Mafra D, Borges N, Alvarenga L, Esgalhado M, Cardozo L, Lindholm B, et al. Dietary components that may influence the disturbed gut microbiota in chronic kidney disease. Nutrients. 2019;11:496.

39. Vaziri ND, Zhao YY, Pahl MV. Altered intestinal microbial flora and impaired epithelial barrier structure and function in CKD: the nature, mechanisms, consequences and potential treatment. Nephrol Dial Transplant. 2016;31: 737-46

40. Castillo-Rodriguez E, Fernandez-Prado R, Esteras R, Perez-Gomez MV, GraciaIguacel C, Fernandez-Fernandez B, et al. Impact of altered intestinal microbiota on chronic kidney disease progression. Toxins (Basel). 2018;10:300.

41. Rios-Covian D, Ruas-Madiedo P, Margolles A, Gueimonde M, de Los ReyesGavilan CG, Salazar N. Intestinal short chain fatty acids and their link with diet and human health. Front Microbiol. 2016;7:185.

42. Tang WH, Wang Z, Kennedy DJ, Wu Y, Buffa JA, Agatisa-Boyle B, et al. Gut microbiota-dependent trimethylamine $\mathrm{N}$-oxide (TMAO) pathway contributes to both development of renal insufficiency and mortality risk in chronic kidney disease. Circ Res. 2015;116:448-55.

43. Lin CJ, Wu V, Wu PC, Wu CJ. Meta-analysis of the associations of p-cresyl sulfate (PCS) and indoxyl sulfate (IS) with cardiovascular events and all-cause mortality in patients with chronic renal failure. PLoS One. 2015;10:e0132589.

44. Akchurin OM, Kaskel F. Update on inflammation in chronic kidney disease. Blood Purif. 2015;39:84-92.

45. De Angelis M, Montemurno E, Piccolo M, Vannini L, Lauriero G, Maranzano $V$, et al. Microbiota and metabolome associated with immunoglobulin A nephropathy (IgAN). PLoS One. 2014;9:e99006.

46. Xiao J, Peng Z, Liao Y, Sun H, Chen W, Chen X, et al. Organ transplantation and gut microbiota: current reviews and future challenges. Am J Transl Res. 2018;10:3330-44.

47. Johansen KL, Chertow GM, Ng AV, Mulligan K, Carey S, Schoenfeld PY, et al. Physical activity levels in patients on hemodialysis and healthy sedentary controls. Kidney Int. 2000;57:2564-70.

48. Zamojska S, Szklarek M, Niewodniczy M, Nowicki M. Correlates of habitual physical activity in chronic haemodialysis patients. Nephrol Dial Transplant. 2006;21:1323-7.

49. Maleki D, Locke GR III, Camilleri M, Zinsmeister AR, Yawn BP, Leibson C, et al. Gastrointestinal tract symptoms among persons with diabetes mellitus in the community. Arch Intern Med. 2000;160:2808-16.

50. Sommers T, Mitsuhashi S, Singh P, Hirsch W, Katon J, Ballou S, et al. Prevalence of chronic constipation and chronic diarrhea in diabetic individuals in the United States. Am J Gastroenterol. 2019;114:135-42.

51. Yamada E, Namiki Y, Takano Y, Takamine H, Inazumi K, Sasaki H, et al. Clinical factors associated with the symptoms of constipation in patients with diabetes mellitus: a multicenter study. J Gastroenterol Hepatol. 2018;33:863-8.

52. Pepe J, Badiali D, Biviano I, Nofroni I, Romagnoli E, Cilli M, et al. The effect of parathyroidectomy on chronic constipation in patients affected by primary hyperparathyroidism. J Bone Miner Metab. 2013;31:690-4.

53. Ogata H, Koiwa F, Shishido K, Kinugasa E. Combination therapy with sevelamer hydrochloride and calcium carbonate in Japanese patients with long-term hemodialysis: alternative approach for optimal mineral management. Ther Apher Dial. 2005;9:11-5.

54. Braunlin W, Zhorov E, Guo A, Apruzzese W, Xu Q, Hook P, et al. Bile acid binding to sevelamer $\mathrm{HCl}$. Kidney Int. 2002;62:611-9.

55. Ikee R. Cardiovascular disease, mortality, and magnesium in chronic kidney disease: growing interest in magnesium-related interventions. Ren Replace Ther. 2018:4:1

56. Wald A. Bile acids and bowel function: do they play a role in constipation-associated irritable bowel syndrome? Clin Gastroenterol Hepatol. 2018;16:486-7.

57. Floege J, Covic AC, Ketteler M, Rastogi A, Chong EM, Gaillard S, et al. A phase III study of the efficacy and safety of a novel iron-based phosphate binder in dialysis patients. Kidney Int. 2014;86:638-47.

58. Yokoyama K, Akiba T, Fukagawa M, Nakayama M, Sawada K, Kumagai Y, et al. A randomized trial of JTT-751 versus sevelamer hydrochloride in patients on hemodialysis. Nephrol Dial Transplant. 2014;29:1053-60.

59. Kortman GA, Dutilh BE, Maathuis AJ, Engelke UF, Boekhorst J, Keegan KP, et al. Microbial metabolism shifts towards an adverse profile with supplementary iron in the TIM-2 in vitro model of the human colon. Front Microbiol. 2016;6:1481

60. Lee T, Clavel T, Smirnov K, Schmidt A, Lagkouvardos I, Walker A, et al. Oral versus intravenous iron replacement therapy distinctly alters the gut microbiota and metabolome in patients with IBD. Gut. 2017:66:863-71.

61. Lau WL, Vaziri ND, Nunes AC, Comeau AM, Langille MG, England W, et al. The phosphate binder ferric citrate alters the gut microbiome in rats with chronic kidney disease. J Pharmacol Exp Ther. 2018;367:452-60.

62. Khoueiry G, Waked A, Goldman M, El-Charabaty E, Dunne E, Smith M, et al. Dietary intake in hemodialysis patients does not reflect a heart healthy diet. J Ren Nutr. 2011;21:438-47.

63. Bovio G, Esposito C, Montagna G, Brazzo S, Esposito V, Torreggiani M, et al. Inadequate macronutrient and micronutrient intakes in hemodialysis and peritoneal dialysis patients: data from a seven-day weighed dietary record. Nephron. 2016;133:253-60.

64. Luis D, Zlatkis K, Comenge B, Garcia Z, Navarro JF, Lorenzo V, et al. Dietary quality and adherence to dietary recommendations in patients undergoing hemodialysis. J Ren Nutr. 2016:26:190-5.

65. Demirci BG, Tutal E, Eminsoy IO, Kulah E, Sezer S. Dietary fiber intake: its relation with glycation end products and arterial stiffness in end-stage renal disease patients. J Ren Nutr. 2019;29:136-42.

66. Voderholzer WA, Schatke W, Muhldorfer BE, Klauser AG, Birkner B, MullerLissner SA. Clinical response to dietary fiber treatment of chronic constipation. Am J Gastroenterol. 1997;92:95-8.

67. Liu L, Wang S, Liu J. Fiber consumption and all-cause, cardiovascular, and cancer mortalities: a systematic review and meta-analysis of cohort studies. Mol Nutr Food Res. 2015:59:139-46.

68. Cases A, Cigarran-Guldris S, Mas S, Conzalez-Parra E. Vegetable-based diets for chronic kidney disease? It is time to reconsider. Nutrients. 2019;11:1263.

69. Castro-Combs J, Garcia CJ, Majewski M, Wallner G, Sarosiek J. Impaired viscosity of gastric secretion and its mucin content as potential contributing factors to the development of chronic constipation. Dig Dis Sci. 2014;59: 2730-4.

70. Gonzalez A, Krieg R, Massey HD, Carl D, Ghosh S, Gehr TW, et al. Sodium butyrate ameliorates insulin resistance and renal failure in CKD rats by modulating intestinal permeability and mucin expression. Nephrol Dial Transplant. 2019:34:783-94.

71. Chandrasekharan B, Anitha M, Blatt R, Shahnavaz N, Kooby D, Staley C, et al. Colonic motor dysfunction in human diabetes is associated with enteric neuronal loss and increased oxidative stress. Neurogastroenterol Motil. 2011 23:131-8 e26.

72. Kashyap P, Farrugia G. Oxidative stress: key player in gastrointestinal complications of diabetes. Neurogastroenterol Motil. 2011;23:111-4.

73. Cianciolo G, De Pascalis A, Di Lullo L, Ronco C, Zannini C, La Manna G. Folic acid and homocysteine in chronic kidney disease and cardiovascular disease progression: which comes first? Cardiorenal Med. 2017;7:255-66.

74. Givvimani S, Munjal C, Narayanan N, Aqil F, Tyagi G, Metreveli N, et al. Hyperhomocysteinemia decreases intestinal motility leading to constipation. Am J Physiol Gastrointest Liver Physiol. 2012;303:G281-90.

75. Dimidi E, Christodoulides S, Fragkos KC, Scott SM, Whelan K. The effect of probiotics on functional constipation in adults: a systematic review and meta-analysis of randomized controlled trials. Am J Clin Nutr. 2014;100: 1075-84. 
76. Yu T, Zheng YP, Tan JC, Xiong WJ, Wang Y, Lin L. Effects of prebiotics and synbiotics on functional constipation. Am J Med Sci. 2017;353:282-92.

77. Nakabayashi I, Nakamura M, Kawakami K, Ohta T, Kato I, Uchida K, et al. Effects of synbiotic treatment on serum level of p-cresol in haemodialysis patients: a preliminary study. Nephrol Dial Transplant. 2011;26:1094-8

78. Salmean YA, Segal MS, Palii SP, Dahl WJ. Fiber supplementation lowers plasma p-cresol in chronic kidney disease patients. J Ren Nutr. 2015;25: 316-20.

79. McFarlane C, Ramos Cl, Johnson DW, Campbell KL. Prebiotic, probiotic, and synbiotic supplementation in chronic kidney disease: a systematic review and meta-analysis. J Ren Nutr. 2019;29:209-20.

80. Matsuo H, Nakamura K, Nishida A, Kubo K, Nakagawa R, Sumida Y. A case of hypermagnesemia accompanied by hypercalcemia induced by a magnesium laxative in a hemodialysis patient. Nephron. 1995;71: 477-8.

81. Jung GJ, Gil HW, Yang JO, Lee EY, Hong SY. Severe hypermagnesemia causing quadriparesis in a CAPD patient. Perit Dial Int. 2008;28:206.

82. Roerig JL, Steffen KJ, Mitchell JE, Zunker C. Laxative abuse: epidemiology, diagnosis and management. Drugs. 2010;70:1487-503.

83. Wald A. Is chronic use of stimulant laxatives harmful to the colon? J Clin Gastroenterol. 2003;36:386-9.

84. Muller-Lissner SA, Kamm MA, Scarpignato C, Wald A. Myths and misconceptions about chronic constipation. Am J Gastroenterol. 2005;100: 232-42.

85. Black CJ, Burr NE, Quigley EM, Moayyedi P, Houghton LA, Ford AC. Efficacy of secretagogues in patients with irritable bowel syndrome with constipation: systematic review and network meta-analysis. Gastroenterology. 2018;155:1753-63.

86. Nakajima A, Seki M, Taniguchi S, Ohta A, Gillberg PG, Mattsson JP, et al. Safety and efficacy of elobixibat for chronic constipation: results from a randomised, double-blind, placebo-controlled, phase 3 trial and an openlabel, single-arm, phase 3 trial. Lancet Gastroenterol Hepatol. 2018;3:537-47.

87. Gen S, Nobe K, Ikeda N. Lubiprostone, a novel laxative, might improve hyperphosphatemia without water dilution. Ren Replace Ther. 2016;2:50,

88. De Lisle RC. Lubiprostone stimulates small intestinal mucin release. BMC Gastroenterol. 2012:12:156

89. Majewski M, Sarosiek I, Wallner G, Edlavitch SA, Sarosiek J. Stimulation of mucin, mucus, and viscosity during lubiprostone in patients with chronic constipation may potentially lead to increase of lubrication. Clin Transl Gastroenterol. 2014;5:e66.

90. Mishima E, Fukuda S, Shima H, Hirayama A, Akiyama Y, Takeuchi Y, et al. Alteration of the intestinal environment by lubiprostone is associated with amelioration of adenine-induced CKD. J Am Soc Nephrol. 2015;26:1787-94.

91. Moeser AJ, Nighot PK, Roerig B, Ueno R, Blikslager AT. Comparison of the chloride channel activator lubiprostone and the oral laxative Polyethylene Glycol 3350 on mucosal barrier repair in ischemic-injured porcine intestine. World J Gastroenterol. 2008;14:6012-7.

92. Kato T, Honda Y, Kurita Y, Iwasaki A, Sato T, Kessoku T, et al. Lubiprostone improves intestinal permeability in humans, a novel therapy for the leaky gut: a prospective randomized pilot study in healthy volunteers. PLoS One. 2017;12:e0175626.

93. Shin A, Camilleri M, Kolar G, Erwin P, West CP, Murad MH. Systematic review with meta-analysis: highly selective 5-HT4 agonists (prucalopride, velusetrag or naronapride) in chronic constipation. Aliment Pharmacol Ther. 2014;39: 239-53.

94. Gao R, Tao Y, Zhou C, Li J, Wang X, Chen L, et al. Exercise therapy in patients with constipation: a systematic review and meta-analysis of randomized controlled trials. Scand J Gastroenterol. 2019;54:169-77.

95. Clarkson MJ, Bennett PN, Fraser SF, Warmington SA. Exercise interventions for improving objective physical function in patients with end-stage kidney disease on dialysis: a systematic review and meta-analysis. Am J Physiol Renal Physiol. 2019;316:F856-72.

96. Yamagata K, Hoshino J, Sugiyama H, Hanafusa N, Shibagaki Y, Komatsu Y, et al. Clinical practice guideline for renal rehabilitation: systematic reviews and recommendations of exercise therapies in patients with kidney diseases. Ren Replace Ther. 2019:5:28.

97. Bohm C, Schick-Makaroff K, MacRae JM, Tan M, Thompson S. The role of exercise in improving patient-reported outcomes in individuals on dialysis: a scoping review. Semin Dial. 2019;32:336-50.
98. Modi RM, Hinton A, Pinkhas D, Groce R, Meyer MM, Balasubramanian G, et al. Implementation of a defecation posture modification device: impact on bowel movement patterns in healthy subjects. J Clin Gastroenterol. 2019;53:216-9.

99. Sikirov D. Comparison of straining during defecation in three positions: results and implications for human health. Dig Dis Sci. 2003;48:1201-5.

\section{Publisher's Note}

Springer Nature remains neutral with regard to jurisdictional claims in published maps and institutional affiliations.
Ready to submit your research? Choose BMC and benefit from:

- fast, convenient online submission

- thorough peer review by experienced researchers in your field

- rapid publication on acceptance

- support for research data, including large and complex data types

- gold Open Access which fosters wider collaboration and increased citations

- maximum visibility for your research: over $100 \mathrm{M}$ website views per year

At BMC, research is always in progress.

Learn more biomedcentral.com/submissions 